\title{
Existence of Green's Functions for Dilute Bose Gases
}

\author{
M. Duneau and B. Souillard \\ Centre de Physique Théorique de l'Ecole Polytechnique Paris, France \\ «Equipe de Recherche associée au C.N.R.S.»
}

Received January 1, 1973

\begin{abstract}
Some properties of finite volume Green's functions are obtained, and the infinite volume limit is shown to exist for the multi-time Green's functions of a dilute Bose gas, constructed with the operators of the quasi-local algebra (see Theorem IV.5).
\end{abstract}

\section{Introduction and Notations}

For our study of a quantum system of identical bosons, we need:

- A Fock-space $\mathscr{H}_{\Lambda}$, associated to each bounded open set $A$ of the configuration space $\mathbb{R}^{v}: \mathscr{H}_{\Lambda}=\bigoplus_{n \geqq 0} \mathscr{H}_{\Lambda}^{(n)}$, where $\mathscr{H}_{\Lambda}^{(n)}=L_{+}^{2}\left(\Lambda^{n}\right)$ is the Hilbert space of complex square integrable functions with support in $\Lambda^{n}$, symmetric with respect to the arguments.

- A Hamiltonian $H_{\Lambda}$, defined from a two-body potential $\phi$ depending on the relative position $x \in \mathbb{R}^{v}$, and satisfying the following properties:

1) $\phi$ is a real function, continuous outside of the origin (everywhere if $v=1$ ).

2) $\phi$ is stable: $\exists B \geqq 0$ such that $\forall n>0, \forall x_{1}, \ldots, x_{n} \in \mathbb{R}^{v}$ :

$$
U\left(x_{1}, \ldots, x_{n}\right)=\sum_{i<j} \phi\left(x_{\imath}-x_{j}\right) \geqq-n \cdot B .
$$

3) In the end of part III, and in part IV for Theorems 2 and following we need also that $\phi$ is square integrable in the whole space. Then for each $n \geqq 0, H_{A}^{(n)}$, formally equal to $-\mu \cdot n+\sum_{i=1}^{n} \frac{p_{i}^{2}}{2 m}+U\left(x_{1}, \ldots, x_{n}\right)$ is a selfadjoint operator with domain $\mathscr{D}\left(H_{A}^{(n)}\right)$, bounded from below, defined by use of the Wiener integral and the Feynmann-Kac formula [1]. Now an essentially self-adjoint operator is defined by $\sum_{n=0}^{N} \psi^{(n)} \rightarrow \sum_{n=0}^{N} H_{\Lambda}^{(n)} \psi^{(n)}$ where $N<\infty$ and $\psi^{(n)} \in \mathscr{D}\left(H_{\Lambda}^{(n)}\right)$, and the Hamiltonian $H_{\Lambda}$ is the closure of this operator with domain $\mathscr{D}\left(H_{A}\right)=\left\{\psi \in \mathscr{H}_{A}: \sum_{n \geqq 0}\left\|H_{A}^{(n)} \psi^{(n)}\right\|^{2}<\infty\right\}$. 
In the following we assume $\mu+B<0$ in order to apply some results quoted in references, and then $H_{A}^{(n)}$ is a positive operator.

- A density operator on $\mathscr{H}_{\Lambda}$, in the grand-canonical formation for a system of particles in thermal equilibrium in $\Lambda: \varrho_{A}=Z_{\Lambda}^{-1} \exp \left\{-\beta \cdot H_{\Lambda}\right\}$ where $Z_{A}=\operatorname{Tr}_{\mathscr{H}_{A}} \exp \left\{-\beta H_{A}\right\}$ and $\beta$ is the inverse temperature.

- The operators $a(\varphi)$ and $a(\varphi)^{*}$, for any $\varphi \in L^{2}\left(\Lambda_{0}\right), \Lambda_{0} \subseteq \Lambda$. As creation and anihilation operators of bosons are unbounded, it is useful sometimes to work with bounded operators $U(\varphi)$ which define the $C^{*}$-algebra. The operators $U(\varphi)$ are defined in the following way: for any $\varphi \in L^{2}\left(\Lambda_{0}\right), a(\varphi)+a(\varphi)^{*}$ is symmetric and $U(\varphi)$ is the exponential of $i$ times the unique self-adjoint extension. Formally

$$
U(\varphi)=\exp i\left\{a(\varphi)+a(\varphi)^{*}\right\} .
$$

(The operators $U(\varphi)$ define the same $C^{*}$-algebra as the well-known operators $U(\varphi)=e^{i \phi(\varphi)}$ and $V(\varphi)=e^{i \pi(\varphi)}$ with $\varphi$ real.)

We give now some notations in order to define the finite volume Green's functions:

if $x \in \mathbb{R}^{q v}, y \in \mathbb{R}^{r v}$

if $\varphi \in L^{2}\left(\Lambda_{0}^{q}\right), \psi \in L^{2}\left(\Lambda_{0}^{r}\right)$

if $p>0$ and $\sum_{i=1}^{p} q_{l}=\sum_{l=1}^{p} r_{i}=N$ we set $X=\left(x_{1} \ldots x_{p}\right)$ where $x_{i} \in \mathbb{R}^{q_{\imath} v}$

$Y=\left(y_{1} \ldots y_{p}\right)$ where $y_{i} \in \mathbb{R}^{r_{2} v}$

$\Phi=\left(\varphi_{1} \ldots \varphi_{p}\right)$ where $\varphi_{i} \in L^{2}\left(\Lambda_{0}^{q_{t}}\right)$

$\Psi=\left(\psi_{1} \ldots \psi_{p}\right)$ where $\psi_{l} \in L^{2}\left(\Lambda_{0}^{r_{i}}\right)$.

If the chemical potential $\mu$ is low enough, i.e. if the gas is dilute, then the following Green's functions are well defined [2] for

$$
\begin{aligned}
\zeta^{p} \in \mathscr{D}: \mathscr{D} & =\left\{\zeta^{p} \in \mathbb{C}^{p}: \operatorname{Re} \zeta_{1}<\operatorname{Re} \zeta_{2}<\cdots<\operatorname{Re} \zeta_{p}<\beta+\operatorname{Re} \zeta_{1}\right\}, \Lambda_{0} \leqq A, \\
G_{A}\left(X, Y ; \zeta^{p}\right) & =Z_{A}^{-1} \operatorname{Tr}_{\mathscr{H}_{\Lambda}}\left\{A\left(x_{1}, y_{1}\right) e^{-\left(\zeta_{2}-\zeta_{1}\right) H_{A}} \ldots A\left(x_{p}, y_{p}\right) e^{-\left(\beta+\zeta_{1}-\zeta_{p}\right) H_{A}}\right\}, \\
G_{A}\left(\Phi, \Psi ; \zeta^{p}\right) & =Z_{A}^{-1} \operatorname{Tr}_{\mathscr{H}_{A}}\left\{A\left(\varphi_{1}, \psi_{1}\right) e^{-\left(\zeta_{2}-\zeta_{1}\right) H_{A}} \ldots A\left(\varphi_{p}, \psi_{p}\right) e^{-\left(\beta+\zeta_{1}-\zeta_{p}\right) H_{A}}\right\} .
\end{aligned}
$$

Besides Green's functions of creators and anihilators, we shall study Green's functions constructed with the exponential operators generating the local $C^{*}$-algebra. Using these, we work with bounded operators but we loose linearity with respect to test functions.

Let $\varphi \in L^{2}\left(\Lambda_{0}\right), \Lambda_{0} \leqq \Lambda$ and $U(\varphi)=\exp i\left\{a(\varphi)+a(\varphi)^{*}\right\}$. If $p>0$ we set $\Phi=\left(\varphi_{1}, \ldots, \varphi_{p}\right)$ where $\varphi_{i} \in L^{2}\left(\Lambda_{0}\right)$. Then the following Green's functions are well defined for $\zeta^{p} \in \overline{\mathscr{D}}$ (closure of $\left.\mathscr{D}\right)$ :

$$
\mathscr{G}_{A}\left(\Phi ; \zeta^{p}\right)=Z_{A}^{-1} \operatorname{Tr}_{\mathscr{H}_{A}}\left\{U\left(\varphi_{1}\right) e^{-\left(\zeta_{2}-\zeta_{1}\right) H_{A}} \ldots U\left(\varphi_{p}\right) e^{-\left(\beta+\zeta_{1}-\zeta_{p}\right) H_{A}}\right\} .
$$


In Sections I, II we study some properties of finite volume Green's functions of both types $G_{A}\left(\Phi, \Psi ; \zeta^{p}\right)$ and $\mathscr{G}_{A}\left(\Phi ; \zeta^{p}\right)$.

In Sections III, IV we study the corresponding infinite volume limit, in order to give sense to the multitime infinite volume Green's functions.

\section{Finite Volume Green's Functions $\mathrm{G}_{\Lambda}\left(\Phi, \Psi ; \zeta^{p}\right)$}

In this section, we are interested in the existence, continuity and analytical properties of $G_{\Lambda}\left(\Phi, \Psi ; \zeta^{p}\right)$ with respect to $\zeta^{p}$ and in the continuity properties with respect to $\Phi$ and $\Psi$.

Theorem I.1. $G_{A}\left(\Phi, \Psi ; \zeta^{p}\right)$ is an analytic function with respect to $\zeta^{p} \in \mathscr{D}$, which extends to a continuous function on $\overline{\mathscr{D}}$.

We shall see, that using the spectral projectors of the particle-number operators, we can replace first the operators $A(\varphi, \psi)$ by bounded operators on $\mathscr{H}_{A}$. The corresponding result is given in Lemma 1.2 and will be used again in Section II.

Lemma I.2. Let $\mathscr{H}$ be a Hilbert space, $H$ a positive self-adjoint operator such that $\forall \beta>0, Z(\beta)=\operatorname{Tr}\left\{e^{-\beta H}\right\}<\infty$. Let $A_{1}, \ldots, A_{p}$ be bounded operators on $\mathscr{H}$. Then $G\left(\zeta^{p}\right)=Z(\beta)^{-1} \operatorname{Tr}\left\{A_{1} e^{-\left(\zeta_{2}-\zeta_{1}\right) H} \ldots A_{p} e^{-\left(\beta+\zeta_{1}-\zeta_{p}\right) H}\right\}$ is an analytic functions of $\zeta^{p} \in \mathscr{D}$ which extends to a continuous function on $\overline{\mathscr{D}}$.

Proof of I.2. $H$ has a discrete spectrum with finite degeneracy; then $e^{-\hat{\lambda} H}$ is strongly analytic in the half complex plane $\operatorname{Re} \hat{\imath}>0$ and strongly continuous at the boundary. Let $\left\{\psi_{i}\right\}_{i \in I}$ be a basis of eigenvectors of $H$, with $H \psi_{i}=e_{l} \psi_{i}$ and let

$$
G_{i}\left(\zeta^{p}\right)=Z(\beta)^{-1}\left(\psi_{i}, A_{1} e^{-\left(\zeta_{2}-\zeta_{1}\right) H} \ldots e^{-\left(\zeta_{p}-\zeta_{p-1}\right) H} A_{p} \psi_{i}\right) \cdot e^{-\left(\beta+\zeta_{1}-\zeta_{p}\right) e_{2}} .
$$

$G_{i}\left(\zeta^{p}\right)$ is analytic in $\mathscr{D}$ and continuous on $\overline{\mathscr{D}}$ as we see by the two following arguments:

$F_{i}(\hat{\lambda})=\left(\psi_{l}, A_{1} e^{-\lambda_{1} H} \ldots e^{-\lambda_{p-1} \cdot H} A_{p} \psi_{i}\right)$ is analytic with respect to $\lambda=\left(\lambda_{1}, \ldots, \lambda_{p}\right) \in \mathbb{C}^{p-1}$ and $\operatorname{Re} \lambda_{i}>0$. This comes from Hartog's theorem [3].

- $F_{l}(\lambda)$ is continuous for $\operatorname{Re} \lambda_{i} \geqq 0$. In fact:

$F_{i}(\lambda)-F_{i}(\mu)=\sum_{k=1}^{p-1}\left(\psi_{i}, \prod_{j=1}^{k-1} A_{j} e^{-\lambda_{j} H} A_{k}\left[e^{-\lambda_{k} H}-e^{-\mu_{k} H}\right] \prod_{j=k+1}^{p-1} A_{j} e^{-\mu_{j} H} A_{p} \psi_{i}\right)$.

Using Schwartz's inequality and positiveness of $H$, we get:

$$
\left|F_{i}(\lambda)-F_{i}(\mu)\right| \leqq \sum_{h=1}^{p-1} \prod_{j=1}^{n-1}\left\|A_{j}\right\| \cdot\left\|A_{k}\left[e^{-\lambda_{h} H}-e^{-\mu_{k} H}\right] \phi_{i . k}\right\|
$$

where $\phi_{l, k}=\prod_{j=k+1}^{p-1} A_{j} \cdot e^{-\mu, H} A_{p_{l}} \psi_{l}$ depends only of point $\mu$. Continuity of $F_{l}(\lambda)$ at point $\mu$ comes from strong continuity of $e^{-\lambda H}$. 
Now, we have $G\left(\zeta^{p}\right)=\sum_{i \in I} G_{i}\left(\zeta^{p}\right)$ where $G_{i}\left(\zeta^{p}\right)$ is analytic in $\mathscr{D}$ and continuous on $\overline{\mathscr{D}}$. The uniform convergence of the series expansion of $G\left(\zeta^{p}\right)$ on $\overline{\mathscr{D}}$ completes the proof of the lemma:

let

$$
\begin{aligned}
& \mathscr{D}_{k}=\left\{\zeta^{p} \in \overline{\mathscr{D}}: \operatorname{Re}\left(\zeta_{k+1}-\zeta_{k}\right)>\frac{\beta}{2 p}\right\}, \quad k<p \\
& \mathscr{D}_{p}=\left\{\zeta^{p} \in \overline{\mathscr{D}}: \beta+\operatorname{Re}\left(\zeta_{1}-\zeta_{p}\right)>\frac{\beta}{2 p}\right\} .
\end{aligned}
$$

Then $\mathscr{D} C \bigcup_{k=1}^{p} \mathscr{D}_{k}=\overline{\mathscr{D}}$.

We prove now that the expansion of $G\left(\zeta^{p}\right)$ is normally convergent on any $\mathscr{D}_{k}$. For instance, if $\zeta^{p} \in \mathscr{D}_{p}$

and

$$
\left|G_{i}\left(\zeta^{p}\right)\right| \leqq Z(\beta)^{-1} \prod_{j=1}^{p}\left\|A_{j}\right\| e^{-\left(\beta+\operatorname{Re}\left(\zeta_{1}-\zeta_{p}\right)\right) e_{i}} \leqq Z(\beta)^{-1} \prod_{j=1}^{p}\left\|A_{j}\right\| e^{-\frac{\beta}{2 p} e_{i}}
$$

$$
\sum_{i \in I}\left|G_{i}\left(\zeta^{p}\right)\right| \leqq \prod_{j=1}^{p}\left\|A_{j}\right\| Z(\beta)^{-1} \cdot Z\left(\frac{\beta}{2 p}\right)<\infty .
$$

Proof of I.1. Let $E_{n}$ be the projector on $\mathscr{H}_{A}^{(n)}$; formally we get:

$$
\begin{gathered}
G_{\Lambda}\left(\Phi, \Psi ; \zeta^{p}\right) \\
=Z_{A}^{-1} \sum_{n \geqq 0} \operatorname{Tr}_{\mathscr{H}_{A}}\left\{E_{n} A\left(\varphi_{1}, \psi_{1}\right) e^{-\left(\zeta_{2}-\zeta_{1}\right) H_{1}} \ldots A\left(\varphi_{p}, \psi_{p}\right) e^{-\left(\beta+\zeta_{1}-\zeta_{p}\right) H_{A}}\right\} .
\end{gathered}
$$

As $H_{A}$ does not change the number of particles, we can replace in each term of the expansion $A\left(\varphi_{i}, \psi_{l}\right)$ by $E_{(n-N, n+N)} A\left(\varphi_{l}, \psi_{l}\right) E_{(n-N, n+N)}$ which is bounded, and $H_{A}$ by

$$
E_{(n-N, n+N)} H_{\Lambda} \text {, with } E_{(n-N, n+N)}=\sum_{\operatorname{Sup}\{0, n-N\}}^{n+N} E_{j} \text {, and } N=\sum_{i=1}^{p} q_{i}=\sum_{i=1}^{p} r_{l} \text {. }
$$

Using Hölder's inequality [7], we find:

$$
\begin{aligned}
& \operatorname{Tr}_{\mathscr{H}_{A}}\left\{E_{n} A\left(\varphi_{1}, \psi_{1}\right) \cdot e^{-\left(\zeta_{2}-\zeta_{1}\right) H_{A}} \ldots A\left(\varphi_{p}, \psi_{p}\right) e^{-\left(\beta+\zeta_{1}-\zeta_{p}\right) H_{A}}\right\} \mid \\
& \leqq \prod_{i=1}^{p}\left\|E_{(n-N, n+N)} A\left(\varphi_{i}, \psi_{i}\right)\right\| \cdot \operatorname{Tr}_{\mathscr{H}_{A}}\left\{E_{(n-N, n+N)} e^{-\beta H_{A}}\right\} \\
& \leqq \prod_{i=1}^{p}\left\|E_{(n-N, n-N)} A\left(\varphi_{i}, \psi_{i}\right)\right\| \sum_{\operatorname{Sup}\{0, n-N\}}^{n+N} Z_{\Lambda}^{(j)}
\end{aligned}
$$

where $Z_{A}^{(j)}=\operatorname{Tr}_{\mathscr{H}_{A}}\left\{E_{j} \cdot e^{-\beta H_{A}}\right\}$.

It follows from properties of creation and anihilation operators that:

$$
\left\|E_{(n-N, n+N)} A\left(\varphi_{i}, \psi_{i}\right)\right\| \leqq(n+N)^{\frac{q_{i}+r_{i}}{2}}\left\|\varphi_{\imath}\right\| \cdot\left\|\psi_{i}\right\| .
$$


Then

$$
\begin{gathered}
\operatorname{Tr}_{\mathscr{H}_{A}}\left\{E_{n} A\left(\varphi_{1}, \psi_{1}\right) e^{-\left(\zeta_{2}-\zeta_{1}\right) H_{A}} \ldots A\left(\varphi_{p}, \psi_{p}\right) e^{-\left(\beta+\zeta_{1}-\zeta_{p}\right) H_{A}}\right\} \mid \\
\leqq(n+N)^{N} \prod_{i=1}^{p}\left\|\varphi_{i}\right\|\left\|\psi_{i}\right\| \sum_{\text {Sup }\{0, n-N\}}^{n+N} Z_{\Lambda}^{(j)} .
\end{gathered}
$$

As $\mu+B<0, H_{A}$ is positive and by Lemma I.2, we know that each term of the expansion of $G_{A}\left(\Phi, \Psi ; \zeta^{p}\right)$ is analytic in $\mathscr{D}$ and continuous on $\overline{\mathscr{D}}$. We check now that expansion converges normally, i.e.

$$
\sum_{n \geqq 0}(n+N)^{N} \sum_{\operatorname{Sup}\{0, n-N\}}^{n+N} Z_{\Lambda}^{(j)}<\infty .
$$

Let $z=e^{\beta \mu}$ and $Z_{\Lambda}^{(j)}=z^{j} Q_{\Lambda}^{(j)}$. It follows from [4] that the expansion $Z_{\Lambda}(z)=\sum_{j \geqq 0} z^{j} Q_{\Lambda}^{(j)}$ has a non-zero convergence radius. Then $z^{m} \cdot \frac{d^{m}}{d z^{m}} Z_{\Lambda}(z)$ $=\sum_{j \geqq m} j(j-1) \ldots(j-m+1) \cdot z^{j} Q_{\Lambda}^{(j)}$ has the same convergence radius. It is clear then that $\forall N \geqq 0$ and $\forall k$ the expansion $\sum_{j \geqq 0}(j+k)^{N} Z_{\Lambda}^{(j)}(z)$ converges for $|z|$ small enough and the same holds for $\sum_{n \geqq 0}(n+N)^{N} \sum_{\operatorname{Sup}\{0, n-N\}}^{n+N} Z_{\Lambda}^{(j)}(z)$,
concluding the proof.

The following theorem gives a necessary condition to apply the basic tool of Sections III and IV, namely Vitali's theorem, used to prove the existence of the infinite volume limit.

Theorem I.3. $G_{A}\left(\Phi, \Psi ; \zeta^{p}\right)$ is bounded on $\operatorname{Re} \mathscr{D}$ and $\mathscr{M}_{n}$ uniformly with respect to $A$ for $\mu$ low enough. Furthermore the family $G_{\Lambda}\left(\Phi, \Psi ; \zeta^{p}\right)$ for any $\Lambda \supseteqq \Lambda_{0}$, and for any $\zeta^{p} \in \operatorname{Re} \mathscr{D}$ or $\zeta^{p} \in \mathscr{M}_{n}$ is an equicontinuous family of functions of $\Phi$ and $\Psi$.

The set $\mathscr{M}_{n}$ introduced in [5] has the following definition:

$$
\begin{gathered}
\mathscr{M}_{n}=\left\{\zeta^{p} \in \overline{\mathscr{D}}: \operatorname{Re}\left(\zeta_{3}-\zeta_{1}\right) \geqq \frac{\beta}{n}, \operatorname{Re}\left(\zeta_{4}-\zeta_{2}\right) \geqq \frac{\beta}{n}, \ldots\right. \\
\left.\quad \ldots, \operatorname{Re}\left(\beta+\zeta_{1}-\zeta_{p-1}\right) \geqq \frac{\beta}{n}, \operatorname{Re}\left(\beta+\zeta_{2}-\zeta_{p}\right) \geqq \frac{\beta}{n}\right\} .
\end{gathered}
$$

Proof I.3. Formally we have $G_{\Lambda}\left(X, Y ; \zeta^{p}\right)=Z_{A}^{-1} \operatorname{Tr}_{\mathscr{H}_{A}}\left\{A\left(x_{1}, y_{1}\right) e^{-\left(\zeta_{2}-\zeta_{1}\right) H_{A}} \ldots A\left(x_{p}, y_{p}\right) e^{-\left(\beta+\zeta_{1}-\zeta_{p}\right) H_{A}}\right\}$.

It follows from Gruber's results [6, p. 263] that if $\beta^{p} \in \operatorname{Re} \mathscr{D}$ and $\mu$ low enough this kernel defines a bounded operator $G_{A}\left(\beta^{p}\right)$ on $L^{2}\left(\mathbb{R}^{N v}\right)$ such that

$$
\left\|G_{\Lambda}\left(\beta^{p}\right)\right\| \leqq C \cdot k^{N} \cdot N !, \quad \forall \beta^{p} \in \operatorname{Re} \mathscr{D} .
$$


Following Ruelle [5], if $\zeta^{p} \in \mathscr{M}_{n}$ wr split $A\left(\varphi_{1}, \psi_{1}\right) e^{-\left(\zeta_{2}-\zeta_{1}\right) H_{A}}$ . ...A $\left(\varphi_{p}, \psi_{p}\right) e^{-\left(\beta+\zeta_{1}-\zeta_{p}\right) H_{A}}$ in a product of operators:

$e^{i t H_{A}} A(\varphi, \psi) e^{-i t H_{A}} \cdot e^{-\frac{\beta}{4 n} H_{A}} ; \quad e^{-\frac{\beta}{4 n} H_{\Lambda}} e^{\imath t H_{A}} A(\varphi, \psi) e^{-\imath t H_{A}}$ and $e^{-\lambda H_{A}}$ with $\operatorname{Re} \lambda \geqq 0$.

Using Hölder's inequality [7], we get:

where

$$
\left|G_{\Lambda}\left(\Phi, \Psi ; \zeta^{p}\right)\right| \leqq \prod_{l=1}^{p}\left|G_{\Lambda}^{(n)}\left(\varphi_{i}, \psi_{\imath}\right)\right|^{\frac{1}{4 n}}
$$

$$
G_{\Lambda}^{(n)}\left(\varphi_{i}, \psi_{l}\right)=\left\{\begin{array}{l}
Z_{\Lambda}^{-1} \operatorname{Tr}_{\mathscr{H}_{A}}\left\{\left[A^{*}\left(\varphi_{i}, \psi_{i}\right) \cdot A\left(\varphi_{i}, \psi_{i}\right) e^{-\frac{\beta}{2 n} H_{A}}\right]^{2 n}\right\} \\
\text { or } \\
Z_{A}^{-1} \operatorname{Tr}_{\mathscr{H}_{A}}\left\{\left[A\left(\varphi_{l}, \psi_{l}\right) \cdot A^{*}\left(\varphi_{i}, \psi_{i}\right) e^{-\frac{\beta}{2 n} H_{A}}\right]^{2 n}\right\} .
\end{array}\right.
$$

Furthermore $\left|G_{A}^{(n)}\left(\varphi_{i}, \psi_{i}\right)\right| \leqq C \cdot k^{2 n\left(q_{2}+r_{2}\right)}\left[2 n\left(q_{i}+r_{i}\right)\right] !\left(\left\|\varphi_{i}\right\| \cdot\left\|\psi_{l}\right\|\right)^{4 n}$ and then

$$
\left|G_{\Lambda}\left(\Phi, \Psi ; \zeta^{p}\right)\right| \leqq C^{\frac{p}{4 n}} k^{N} \prod_{i=1}^{p}\left\{\left[2 n\left(q_{i}+r_{i}\right)\right] !\right\}^{\frac{1}{4 n}}\left\|\varphi_{i}\right\|\left\|\psi_{i}\right\| .
$$

So $G_{\Lambda}\left(\Phi, \Psi ; \zeta^{p}\right)$ is bounded on $\operatorname{Re} \mathscr{D}$ and $\mathscr{M}_{n}$ uniformly in $\Lambda$ and it follows from linearity that the family of $G_{\Lambda}\left(\Phi, \Psi ; \zeta^{p}\right)$ for any $\Lambda \supseteqq \Lambda_{0}$ and any $\zeta^{p} \in \operatorname{Re} \mathscr{D}$ or $\zeta^{p} \in \mathscr{U}_{n}$ is equicontinuous with respect to $\Phi$ and $\Psi$.

\section{Finite Volume Green's Functions $\mathscr{G}_{A}\left(\Phi ; \zeta^{p}\right)$}

We shall get for Green's functions of exponential results similar to those in Section I for Green's functions of creators and anihilators.

Theorem II.1. $\mathscr{G}_{A}\left(\Phi ; \zeta^{p}\right)$ is an analytic function with respect to $\zeta^{p} \in \mathscr{D}$ which extends to a continuous function on $\overline{\mathscr{D}}$, bounded by 1 .

Proof. This follows readily from Lemma I.2 and $\|U(\varphi)\|=1$, $\forall \varphi \in L^{2}\left(\Lambda_{0}\right)$.

The proof of equicontinuity with respect to $\Phi$ requires some more technical computation. We first prove the following theorem:

Theorem II.2. The family $\mathscr{G}_{\Lambda}\left(\Phi ; \zeta^{p}\right)$ for any $\Lambda \supseteqq \Lambda_{0}$ and any $\beta^{p} \in \operatorname{Re} \mathscr{L}$ is an equicontinuous family of functions with respect to $\Phi$, when $\mu$ is low enough.

Proof. Using the formal expansion $U(\varphi)=e^{i a(\varphi)^{*}} \cdot e^{i a(\varphi)} \cdot e^{-\frac{1}{2}\|\varphi\|^{2}}$ we get:

$$
\mathscr{G}_{\Lambda}\left(\Phi ; \zeta^{p}\right)=e^{-\frac{1}{2} \sum_{i=1}^{p}\left\|\varphi_{\imath}\right\|^{2}} \sum_{N \geqq 0} \sum_{q, r}^{(N)} \frac{i^{2 N}}{q ! r !} G_{\Lambda}^{q, r}\left(\Phi ; \zeta^{p}\right)
$$


where $\sum_{q, r}^{(N)}$ runs over all $q=\left(q_{1}, \ldots, q_{p}\right)$ and $r=\left(r_{1}, \ldots, r_{p}\right)$ in $\mathbb{N}^{p}$ such that and

$$
|q|=\sum_{i=1}^{p} q_{i}=|r|=\sum_{i=1}^{p} r_{i}=N ; \quad q !=\prod_{i=1}^{p} q_{i} ; \quad r !=\prod_{i=1}^{p} r_{l}
$$

$$
\begin{aligned}
G_{A}^{q, r}\left(\Phi, \beta^{p}\right)= & Z_{\Lambda}^{-1} \operatorname{Tr}_{\mathscr{H}_{\Lambda}}\left\{a\left(\varphi_{1}\right)^{* q_{1}} a\left(\varphi_{1}\right)^{r_{1}} e^{-\left(\beta_{2}-\beta_{1}\right) H_{\Lambda}} \ldots\right. \\
& \left.\ldots a\left(\varphi_{p}\right)^{* q_{p}} a\left(\varphi_{p}\right)^{r_{p}} e^{-\left(\beta+\beta_{1}-\beta_{p}\right) H_{\Lambda}}\right\}
\end{aligned}
$$

We show now that this expansion of $\mathscr{G}_{\Lambda}\left(\Phi ; \beta^{p}\right)$ is correct.

Firstly, $\mathscr{G}_{\Lambda}\left(\Phi ; \beta^{p}\right)=\sum_{\nu \in \mathbb{N}^{p}} \mathscr{G}_{\Lambda}^{(\nu)}\left(\Phi ; \beta^{p}\right)$ where

$\mathscr{G}_{\Lambda}^{(v)}\left(\Phi ; \beta^{p}\right)=Z_{\Lambda}^{-1} \operatorname{Tr}_{\mathscr{H}_{\Lambda}}\left\{U\left(\varphi_{1}\right) E_{v_{1}} e^{-\left(\beta_{2}-\beta_{1}\right) H_{A}} \ldots U\left(\varphi_{p}\right) E_{v_{p}} e^{-\left(\beta+\beta_{1}-\beta_{p}\right) H_{A}}\right\}$

and $E_{v}$ is the projector over the $v$-particles states. Since $H_{\Lambda} E_{v} \geqq v \cdot \alpha$ with $\alpha=-(\mu+B)>0,\left\|U(\varphi) E_{v} e^{-\lambda H_{A}}\right\| \leqq e^{-\alpha \lambda v}$ and then:

$$
\left|\mathscr{G}_{\Lambda}^{(v)}\left(\Phi ; \beta^{p}\right)\right| \leqq Z_{\Lambda}^{-1} \cdot \prod_{l=1}^{p-1} e^{-x v_{l}\left(\beta_{l+1}-\beta_{l}\right)} \operatorname{Tr}_{\mathscr{H}_{\Lambda}}\left\{E_{v_{p}} e^{-\left(\beta+\beta_{1}-\beta_{p}\right) H_{\Lambda}}\right\} .
$$

So the expression is absolutely convergent and sums up to $\mathscr{G}_{A}\left(\Phi ; \beta^{p}\right)$, because taking the trace and summing up the series commute.

Secondly,

where

$$
\mathscr{G}_{A}^{(v)}\left(\Phi ; \beta^{p}\right)=e^{-\frac{1}{2} \sum_{i=1}^{p}\left\|\varphi_{1}\right\|^{2}} \sum_{q, r \in \mathbb{N}^{p}} \frac{i^{|q+r|}}{q ! r !} G_{A}^{(v, q, r)}\left(\Phi ; \beta^{p}\right)
$$

$$
\begin{aligned}
\mathscr{G}_{\Lambda}^{(v, q, r)}\left(\Phi, \beta^{p}\right)= & Z_{\Lambda}^{-1} \operatorname{Tr}_{\mathscr{H}_{\Lambda}}\left\{a\left(\varphi_{1}\right)^{* q_{1}} a\left(\varphi_{1}\right)^{r_{1}} E_{\nu_{1}} e^{-\left(\beta_{2}-\beta_{1}\right) H_{A}} \ldots\right. \\
& \left.\ldots E_{v_{p}} e^{-\left(\beta+\beta_{1}-\beta_{p}\right) H_{A}}\right\}
\end{aligned}
$$

and we shall show that this series is absolutely convergent. In view of that, we consider the definition of $U(\varphi)$ :

$$
U(\varphi) E_{v}=\sum_{k \in \mathbb{N}} \frac{i^{k}}{k !}\left\{a(\varphi)^{*}+a(\varphi)\right\}^{k} \cdot E_{v}
$$

a normally convergent series.

Using normal reordering, we get:

$$
U(\varphi) E_{v}=\sum_{k \in \mathbb{N}} \frac{i^{k}}{k !} \sum_{q, r \in \mathbb{N}} \Gamma_{q, r}^{k} a(\varphi)^{* q} a(\varphi)^{r} E_{v}
$$

where $\Gamma_{q, r}^{k}$ arises from combinatorics and contractions. This new expansion is also norm convergent when taken account of ${ }^{1} \sum_{k} \frac{\Gamma_{q, r}^{k}}{k !}=\frac{e^{-\frac{1}{2}\|\varphi\|_{2}}}{q ! r !}$ and norm estimates of creators and anihilators. Resuming in $k$ we get

$$
U(\varphi) E_{v}=e^{-\frac{1}{2}\|\varphi\|_{2}} \sum_{q, r \in \mathbb{N}} \frac{i^{q+r}}{q ! r !} a(\varphi)^{* q} a(\varphi)^{r} E_{v}
$$

1 This comes out from the well-known algebraic equality: $e^{X} e^{Y}=e^{X+Y+\frac{1}{2}[X, Y]}$ if $[X, Y] \in \mathbb{C}$. 
which is also norm convergent and then we can commute the summation and the trace, and this ends the second part of our proof.

Thirdly we look at the series $\sum_{v, q, r} \frac{i^{|q+r|}}{q ! r !} G_{\Lambda}^{(v, q, r)}\left(\Phi ; \beta^{p}\right)$. We shall show here that this expansion is absolutely convergent and we shall resume it up. $G_{A}^{(v, q, r)}\left(\Phi, \beta^{p}\right)$ is a finite sum of terms of Gruber's closed loop expansion (cf. [6], p. 253). A bound of the absolute value of each of those terms gives us the following estimate:

Then:

$$
\sum_{v}\left|G_{\Lambda}^{(v, q, r)}\left(\Phi ; \beta^{p}\right)\right| \leqq A(\Lambda) N ! B^{N} \prod_{i=1}^{p}\left\|\varphi_{\imath}\right\|^{q_{\imath}+r_{2}} .
$$

$$
\begin{aligned}
\sum_{q, r} \frac{1}{q ! r !} \sum_{\nu}\left|G_{A}^{(v, q, r)}\left(\Phi ; \beta^{p}\right)\right| & \leqq A(\Lambda) \sum_{N \geqq 0} \frac{B^{N}}{N !}\left(\sum_{i=1}^{p}\left\|\varphi_{\imath}\right\|\right)^{2 N} \\
& =A(\Lambda) \exp \left\{B\left(\sum_{i=1}^{p}\left\|\varphi_{\imath}\right\|\right)^{2}\right\}
\end{aligned}
$$

The expansion being absolutely convergent, we have now:

$$
\sum_{v} \sum_{q, r} \frac{i^{|q+r|}}{q ! r !} G_{\Lambda}^{(v, q, r)}\left(\Phi ; \beta^{p}\right)=\sum_{N \geqq 0} \sum_{q, r}^{(N)} \frac{i^{2 N}}{q ! r !} G_{\Lambda}^{q, r}\left(\Phi ; \beta^{p}\right)
$$

and this ends the proof of the expression

$$
\mathscr{G}_{\Lambda}\left(\Phi ; \beta^{p}\right)=e^{-\frac{1}{2} \sum_{i=1}^{p}\left\|\varphi_{i}\right\|^{2}} \sum_{N \geqq 0} \sum_{q, r}^{(N)} \frac{i^{2 N}}{q ! r !} G_{\Lambda}^{q, r}\left(\Phi ; \beta^{p}\right) .
$$

Now we can use another bound of $G_{A}^{q, r}\left(\Phi ; \beta^{p}\right)$, which is independent of $\Lambda:\left|G_{A}^{q_{1} r}\left(\Phi ; \beta^{p}\right)\right| \leqq C \cdot N ! K^{N} \prod_{i=1}^{p}\left\|\varphi_{\imath}\right\|^{q_{i}+r_{t}}$ (cf. [6], p. 263). This proves that our expansion of $\mathscr{G}_{\Lambda}\left(\Phi ; \beta^{p}\right)$ is uniformly convergent with respect to $A$ and $\beta^{p}$ and then equicontinuity of $G_{A}^{q, r}\left(\Phi ; \beta^{p}\right)$ with respect to $\Phi$, for $\Lambda \supseteqq \Lambda_{0}$ and $\beta^{p} \in \operatorname{Re} \mathscr{D}$ implies the same property for $\mathscr{G}_{\Lambda}\left(\Phi ; \beta^{p}\right)$. Q.E.D.

Now we can extend the continuity in $\Phi$ for any $\zeta^{p} \in \mathscr{M}_{n}$ by use of Hölder's inequality:

Theorem II.3. The family $\mathscr{G}_{\Lambda}\left(\Phi ; \zeta^{p}\right)$ for any $\Lambda \supseteqq \Lambda_{0}$ and any $\zeta^{p} \in \mathscr{M}_{n}$ is equicontinuous with respect to $\Phi$.

Proof. Let $\Phi=\left(\varphi_{1}, \ldots, \varphi_{p}\right)$ and $\Phi^{\prime}=\left(\varphi_{1}^{\prime}, \ldots, \varphi_{p}^{\prime}\right)$

$$
\begin{gathered}
\left|\mathscr{G}_{\Lambda}\left(\Phi^{\prime} ; \zeta^{p}\right)-\mathscr{G}_{\Lambda}\left(\Phi ; \zeta^{p}\right)\right| \\
\leqq \sum_{i=1}^{p}\left|\mathscr{G}_{\Lambda}\left(\varphi_{1}, \ldots, \varphi_{i-1}, \varphi_{l}^{\prime}, \ldots, \varphi_{p}^{\prime}\right)-\mathscr{G}_{\Lambda}\left(\varphi_{1}, \ldots, \varphi_{l-1}, \varphi_{l}, \varphi_{i+1}^{\prime}, \ldots, \varphi_{p}^{\prime}\right)\right| .
\end{gathered}
$$


Using Hölder's inequality, we get:

$$
\begin{gathered}
\left|\mathscr{G}_{\Lambda}\left(\Phi^{\prime} ; \zeta^{p}\right)-\mathscr{G}_{\Lambda}\left(\Phi ; \zeta^{p}\right)\right| \\
\leqq \sum_{l=1}^{p} \prod_{j<1}\left|\mathscr{G}_{\Lambda}^{(n)}\left(\varphi_{j}\right)\right|^{\frac{1}{4 n}} \prod_{j>i}\left|\mathscr{G}_{\Lambda}^{(n)}\left(\varphi_{j}^{\prime}\right)\right|^{\frac{1}{4^{n}}}\left|\mathscr{G}_{\Lambda}^{(n)}\left(\varphi_{l}^{\prime}, \varphi_{l}\right)\right|^{\frac{1}{4 n}}=\sum_{i=1}^{p}\left|\mathscr{G}_{\Lambda}\left(\varphi_{i}^{\prime}, \varphi_{l}\right)\right|^{\frac{1}{4 n}}
\end{gathered}
$$

where

$$
\begin{aligned}
& \mathscr{G}_{\Lambda}^{(n)}\left(\varphi_{j}\right)=\left\{\begin{array}{l}
Z_{\Lambda}^{-1} \operatorname{Tr}_{\mathscr{H}_{A}}\left\{\left[U\left(\varphi_{j}\right) * U\left(\varphi_{j}\right) e^{-\frac{\beta}{2 n} H_{A}}\right]^{2 n}\right\}=1 \\
\operatorname{or}_{\Lambda}^{-1} \operatorname{Tr}_{\mathscr{H}_{\Lambda}}\left\{\left[U\left(\varphi_{j}\right) U\left(\varphi_{j}\right)^{*} e^{-\frac{\beta}{2 n} H_{\Lambda}}\right]^{2 n}\right\}=1,
\end{array}\right. \\
& \mathscr{G}_{\Lambda}^{(n)}\left(\varphi_{i}^{\prime}, \varphi_{i}\right)=\left\{\begin{array}{l}
Z_{\Lambda}^{-1} \operatorname{Tr}_{\mathscr{H}_{\Lambda}}\left\{\left(\left[U\left(\varphi_{i}^{\prime}\right)-U\left(\varphi_{i}\right)\right]^{*}\left[U\left(\varphi_{i}^{\prime}\right)-U\left(\varphi_{i}\right)\right] e^{\left.-\frac{\beta}{2 n} H_{\Lambda}\right)^{2 n}}\right\}\right. \\
\text { or } \\
Z_{\Lambda}^{-1} \operatorname{Tr}_{\mathscr{H}_{\Lambda}}\left\{\left(\left[U\left(\varphi_{i}^{\prime}\right)-U\left(\varphi_{i}\right)\right]\left[U\left(\varphi_{i}^{\prime}\right)-U\left(\varphi_{i}\right)\right]^{*} e^{-\frac{\beta}{2 n} H_{\Lambda}}\right)^{2 n}\right\}
\end{array}\right.
\end{aligned}
$$

and the result follows from Theorem II.2.

\section{Infinite Volume Limit of Green's Functions $\mathrm{G}_{\Lambda}\left(\Phi, \Psi ; \zeta^{p}\right)$}

In this section, we merely recall some results concerning the infinite volume limit of Green's functions $G_{\Lambda}\left(\Phi, \Psi ; \zeta^{p}\right)$. For details, see Ginibre [1], Gruber [6], Ruelle [2].

If the chemical potential $\mu$ is low enough, the following limit exists:

$$
G\left(\Phi, \Psi ; \zeta^{p}\right)=\lim _{\Lambda \rightarrow \infty} G_{\Lambda}\left(\Phi, \Psi ; \zeta^{p}\right) \quad \forall \zeta^{p} \in \mathscr{D} .
$$

The convergence is uniform on the compacts of $\mathscr{D}$ and thus, $G\left(\Phi, \Psi ; \zeta^{p}\right)$ is analytic in $\mathscr{D}$.

The multitime Green's functions are obtained for $\zeta^{p}$ on the distinguished boundary of $\mathscr{D}$, and the problem is to prove the convergence of $G_{A}\left(\Phi, \Psi ; \zeta^{p}\right)$ on that part of $\overline{\mathscr{D}}$, and possibly to check some property of continuity, as those obtained for finite volume.

Actually for Fermi systems, Ruelle has recently proved [5] that the limit Green's function is continuous on $\overline{\mathscr{D}}$.

For a dilute Bose gas, we have the following result: $G\left(\Phi, \Psi ; \zeta^{p}\right)$ extends to a continuous function on $\mathscr{M}_{n}$ and

$$
G\left(\Phi, \Psi ; \zeta^{p}\right)=\lim _{\Lambda \rightarrow \infty} G_{A}\left(\Phi, \Psi ; \zeta^{p}\right), \quad \forall \zeta^{p} \in \mathscr{M}_{n},
$$

the convergence being uniform on the compacts of $\mathscr{M}_{n}$. 
This follows from the uniform boundedness of $\frac{\partial G_{A}}{\partial \zeta_{k}}\left(\Phi, \Psi ; \zeta^{p}\right)$ for each $A$ and $\zeta^{p} \in \mathscr{M}_{n}$; the demonstration uses previous Theorem I.3 and Lemma 1 in Ruelle [5]. This needs the stronger condition that the potential is in $L^{1}\left(\mathbb{R}^{v}\right) \cap L^{2}\left(\mathbb{R}^{v}\right)$.

\section{Infinite Volume Limit of Green's Functions $\mathscr{G}_{A}\left(\Phi ; \zeta^{p}\right)$}

The first result of this section follows from a theorem on functions of complex variables, and shows that the infinite volume limit of $\mathscr{G}_{A}\left(\Phi, \zeta^{p}\right)$ exists and is analytic in $\mathscr{D}$. However the continuity of $\mathscr{G}_{A}\left(\Phi, \zeta^{p}\right)$ on $\overline{\mathscr{D}}$ is so far, of no use, for the infinite volume limit may not exist on the boundary of $\mathscr{D}$, and even if it exists, may not extend continuously the limit in $\mathscr{D}$.

Actually the continuity will follow from a stronger assumption on the interaction: we shall assume that the potential function is not only in $L^{1}\left(\mathbb{R}^{v}\right)$ as before but also in $L^{2}\left(\mathbb{R}^{v}\right)$. Then there exists a uniform bound on the derivatives $\frac{\partial \mathscr{G}_{\Lambda}}{\partial \zeta_{k}}\left(\Phi ; \zeta^{p}\right)$ for any $\Lambda, \zeta^{p} \in \mathscr{M}_{n}$ and particular $\Phi$. The general result will use the equicontinuity proved in Section II.

Theorem IV.1. If the chemical potential is low enough, the following limit exists for each

$$
\zeta^{p} \in \mathscr{D}: \mathscr{G}\left(\Phi ; \zeta^{p}\right)=\lim _{\Lambda \rightarrow \infty} \mathscr{G}_{\Lambda}\left(\Phi, \zeta^{p}\right) .
$$

Furthermore the convergence is uniform on the compacts of $\mathscr{D}$ and $\mathscr{G}\left(\Phi ; \zeta^{p}\right)$ is analytic in $\mathscr{D}$.

Proof. For $\beta^{p} \in \operatorname{Re} \mathscr{D}$ we have the expansion as in Section II:

$$
\mathscr{G}_{\Lambda}\left(\Phi ; \beta^{p}\right)=e^{-\frac{1}{2} \sum_{i=1}^{p}\left\|\varphi_{i}\right\|^{2}} \sum_{N \geqq 0} \sum_{q, r}^{(N)} \frac{i^{2 N}}{q ! r !} G_{A}^{q, r}\left(\Phi ; \beta^{p}\right) .
$$

We know from [1] and [6] that the following limit exists for any

$$
\beta^{p} \in \operatorname{Re} \mathscr{D}: G^{q, r}\left(\Phi ; \beta^{p}\right)=\lim _{A \rightarrow \infty} G_{A}^{q, r}\left(\Phi ; \beta^{p}\right) .
$$

The absolute convergence of the expansion on $\operatorname{Re} \mathscr{D}$ implies the existence of

$$
\mathscr{G}\left(\Phi ; \beta^{p}\right)=\lim _{\Lambda \rightarrow \infty} \mathscr{G}_{\Lambda}\left(\Phi ; \beta^{p}\right) .
$$

Then it follows from Vitali's theorem that

$$
\mathscr{G}\left(\Phi ; \zeta^{p}\right)=\lim _{\Lambda \rightarrow \infty} \mathscr{G}_{\Lambda}\left(\Phi ; \zeta^{p}\right)
$$

exists for any $\zeta^{p} \in \mathscr{D}$; furthermore the convergence is uniform on the compact of $\mathscr{D}$ and $\mathscr{G}\left(\Phi ; \zeta^{p}\right)$ is analytic in $\mathscr{D}$. The next result concerns the derivatives of $\mathscr{G}_{A}\left(\Phi, \zeta^{p}\right)$ for $\zeta^{p} \in \mathscr{M}_{n}$. 
Lemma IV.2. Assume that the potential function belongs to $L^{1}\left(\mathbb{R}^{v}\right)$ $\cap L^{2}\left(\mathbb{R}^{v}\right)$. Let $\Phi=\left(\varphi_{1}, \ldots, \varphi_{p}\right)$ where $\varphi_{i}$ is of class $C^{2}$ with support in $\Lambda_{0}$. Then the derivatives $\frac{\partial \mathscr{G}_{\Lambda}}{\partial \zeta_{k}}\left(\Phi ; \zeta^{p}\right)$ are bounded on $\mathscr{M}_{n}$ uniformly in $\Lambda$.

The proof is similar to the Ruelle's paper one [5].

Proof. We have, using the technique of Lemma I.2:

$$
\begin{aligned}
\frac{\partial \mathscr{G}_{A}}{\partial \zeta_{k}}\left(\Phi ; \zeta^{p}\right)= & Z_{A}^{-1} \operatorname{Tr}_{\mathscr{H}_{A}}\left\{U\left(\varphi_{1}\right) e^{-\left(\zeta_{2}-\zeta_{1}\right) H_{A}} \ldots e^{-\left(\zeta_{k}-\zeta_{k-1}\right) H_{A}}\left[U\left(\varphi_{k}\right), H_{A}\right]\right. \\
& \left.\cdot e^{-\left(\zeta_{k+1}-\zeta_{k}\right) H_{A}} \ldots\right\} .
\end{aligned}
$$

Then using Hölder's inequality for $\zeta^{p} \in \mathscr{M}_{n}$

$$
\left|\frac{\partial \mathscr{G}_{A}}{\partial \zeta_{k}}\left(\Phi: \zeta^{p}\right)\right| \leqq \prod_{i \neq k}\left|\mathscr{G}_{\Lambda}^{(n)}\left(\varphi_{i}\right)\right|^{\frac{1}{4 n}} \cdot\left|\mathscr{G}_{A}^{\prime(n)}\left(\varphi_{k}\right)\right|^{\frac{1}{4 n}}=\left|\mathscr{G}_{A}^{(n)}\left(\varphi_{k}\right)\right|^{\frac{1}{4 n}}
$$

where

and

$$
\mathscr{G}_{\Lambda}^{(n)}\left(\varphi_{i}\right)=\left\{\begin{array}{l}
Z_{A}^{-1} \operatorname{Tr}_{\mathscr{H}_{A}}\left\{\left[U\left(\varphi_{i}\right)^{*} U\left(\varphi_{l}\right) e^{-\frac{\beta}{2 n} H_{A}}\right]^{2 n}\right\}=1 \\
\operatorname{or} Z_{A}^{-1} \operatorname{Tr}_{\mathscr{H}_{A}}\left\{\left[U\left(\varphi_{l}\right) U\left(\varphi_{l}\right)^{*} e^{\left.-\frac{\beta}{2 n} H_{A}\right]^{2 n}}\right\}=1\right.
\end{array}\right.
$$

Now

$$
\mathscr{G}_{\Lambda}^{\prime(n)}\left(\varphi_{k}\right)=\left\{\begin{array}{l}
Z_{\Lambda}^{-1} \operatorname{Tr}_{\mathscr{H}_{A}}\left\{\left(\left[U\left(\varphi_{k}\right), H_{A}\right]^{*}\left[U\left(\varphi_{k}\right), H_{A}\right] e^{-\frac{\beta}{2 n} H_{\Lambda}}\right)^{2 n}\right\} \\
\operatorname{or} \\
Z_{A}^{-1} \operatorname{Tr}_{\mathscr{H}_{A}}\left\{\left(\left[U\left(\varphi_{k}\right), H_{A}\right]\left[U\left(\varphi_{k}\right), H_{A}\right]^{*} e^{-\frac{\beta}{2 n} H_{A}}\right)^{2 n}\right\} .
\end{array}\right.
$$

where

$$
\left[U(\varphi), H_{A}\right]=\left\{\sum_{n \geqq 1} \frac{i^{n}}{n !}\left[a(\varphi)^{*}+a(\varphi), H_{A}\right]^{(n)}\right\} U(\varphi)
$$

$$
\begin{aligned}
& {[X, Y]^{(1)}=[X, Y],} \\
& {[X, Y]^{(n)}=\left[X,[X, Y]^{(n-1)}\right] .}
\end{aligned}
$$

As the Hamiltonian is given by a two-body interaction, we check easily that the expansion of $\left[U(\varphi), H_{A}\right]$ ends at $n=4$ besides, using the commutation relations to obtain the Wick ordering of creation and anihilation operators, we get contractions involving either the free part of $H_{A}$, or the interaction part. The result is an expression of creation and anihilation operators smeared with absolutely integrable functions, or is a constant.

Now we use the fact $([6]$, p. 270$)$ that the kernels $G_{A}\left(X, Y ; \beta^{p}\right)$ defined by:

$$
G_{\Lambda}\left(\Phi, \Psi ; \beta^{p}\right)=\int d X d Y G_{\Lambda}\left(X, Y ; \beta^{p}\right) \Phi(X) \psi(Y)
$$

are bounded functions of $X, Y$, uniformly in $\Lambda$, when $\beta^{p} \in \operatorname{Re} \mathscr{D}$ is fixed. 
It follows then that $\mathscr{G}_{A}^{\prime(n)}\left(\varphi_{k}\right)$ is bounded uniformly in $\Lambda$, and this proves the lemma.

The uniform convergence of $\mathscr{G}_{A}\left(\Phi, \zeta^{p}\right)$ on compacts of $\mathscr{D}$, and the preceeding lemma yield that $\mathscr{G}_{\Lambda}\left(\Phi, \zeta^{p}\right)$ converges on $\mathscr{M}_{n}$, but we can get a stronger result.

Lemma IV.3. Let the potential in $L^{1}\left(\mathbb{R}^{v}\right) \cap L^{2}\left(\mathbb{R}^{v}\right)$. Then $\mathscr{G}\left(\Phi ; \zeta^{p}\right)$ defined on $\mathscr{D}$ extends to a continuous function on $\mathscr{M}_{n} ;$ furthermore $\mathscr{G}\left(\Phi ; \zeta^{p}\right)$ is continuous with respect to $\Phi$, uniformly in $\zeta^{p} \in \mathscr{M}_{n}$ and $\mathscr{G}\left(\Phi ; \zeta^{p}\right)$ $=\lim _{\Lambda \rightarrow \infty} \mathscr{G}_{\Lambda}\left(\Phi ; \zeta^{p}\right)$ uniformly on compacts of $\mathscr{M}_{n}$.

Proof. We first prove the lemma for $\Phi=\left(\varphi_{1}, \ldots, \varphi_{p}\right), \varphi_{2} \in C^{2}$ with support in $\Lambda_{0}$. Let $K$ be any compact of $\mathscr{M}_{n}$; then for any $\alpha>0$ there exists a compact $K_{\alpha}$ of $\mathscr{D} \cap \mathscr{M}_{n}$ such that $\forall \zeta^{p} \in K, \exists \zeta^{\prime p} \in K_{\alpha}:\left|\zeta^{p}-\zeta^{\prime p}\right|<\alpha$ where we use for instance the euclidean distance. Then if $\zeta^{p} \in K$ and $\zeta^{\prime p} \in K_{\alpha}$ with $\left|\zeta^{p}-\zeta^{\prime p}\right|<\alpha$

$$
\begin{aligned}
\left|\mathscr{G}_{\Lambda}\left(\Phi ; \zeta^{p}\right)-\mathscr{G}_{\Lambda^{\prime}}\left(\Phi ; \zeta^{p}\right)\right| \leqq & \left|\mathscr{G}_{\Lambda}\left(\Phi ; \zeta^{p}\right)-\mathscr{G}_{\Lambda}\left(\Phi ; \zeta^{p}\right)\right|+\left|\mathscr{G}_{\Lambda}\left(\Phi ; \zeta^{\prime p}\right)-\mathscr{G}_{\Lambda^{\prime}}\left(\Phi ; \zeta^{p}\right)\right| \\
& +\left|\mathscr{G}_{\Lambda^{\prime}}\left(\Phi ; \zeta^{\prime p}\right)-\mathscr{G}_{\Lambda^{\prime}}\left(\Phi ; \zeta^{p}\right)\right| .
\end{aligned}
$$

The first and third terms are bounded by $C \cdot \alpha$ uniformly in $A$ where $C$ is a bound on the derivatives of $\mathscr{G}_{A}\left(\Phi ; \zeta^{p}\right)$. The second term is arbitrarily small for any $\zeta^{\prime p} \in K_{\alpha}$ if $\Lambda$ and $\Lambda^{\prime}$ are large enough. Thus there exists $\mathscr{G}\left(\Phi ; \zeta^{p}\right)=\lim _{\Lambda \rightarrow \infty} \mathscr{G}_{\Lambda}\left(\Phi ; \zeta^{p}\right)$ uniformly on compacts of $\mathscr{M}_{n}$; the continuity of the limit in $\mathscr{M}_{n}$ follows from Theorem II.1.

Now using Theorem II.3, we can extend these results to any $\Phi=\left(\varphi_{1}, \ldots, \varphi_{p}\right)$ with $\varphi_{i} \in L^{2}\left(\Lambda_{0}\right)$, for the complex functions $C^{2}$ with support in $\Lambda_{0}$ are dense in $L^{2}\left(\Lambda_{0}\right)$, proving that $\mathscr{G}\left(\Phi ; \zeta^{p}\right)$ is continuous with respect to $\Phi$, uniformly in $\zeta^{p} \in \mathscr{M}_{n}$.

The last problem is to define the limit on $\overline{\mathscr{D}}$ and to check the continuity. We just give the result, the proof being the same as in [5].

Lemma IV.4. Let $\Phi=\left(\varphi_{1}, \ldots, \varphi_{p}\right)$ be given and $\Lambda_{0}$ chosen large enough. We assume that $1 \leqq l \leqq p$, that $K$ is compact in $\mathbb{R}$ and that $\zeta_{l+1}, \ldots, \zeta_{p}$ are such that $0<\operatorname{Re} \zeta_{l+1}<\cdots<\operatorname{Re} \zeta_{p}<\frac{\beta}{2}$.

Given $\varepsilon>0$ there exists $\delta>0$ such that:

$$
\begin{gathered}
\mid \mathscr{G}_{\Lambda}\left(\Phi ;-i \theta_{1},-,-i \theta_{l}, \zeta_{p+1}, \ldots, \zeta_{p}\right) \\
-\mathscr{G}_{\Lambda}\left(\Phi ;-i \theta_{1}, \ldots,-i \theta_{l-1}, \zeta_{l}, \zeta_{l+1}, \ldots, \zeta_{p}\right) \mid<\varepsilon
\end{gathered}
$$

whenever $\Lambda \supseteqq \Lambda_{0}$ and $\theta_{l}, \zeta_{l}$ satisfy $\theta_{l} \in K,\left|\zeta_{l}+i \theta_{l}\right|<\delta, 0<\operatorname{Re} \zeta_{l}<\operatorname{Re} \zeta_{l+1}$. Furthermore $\mathscr{G}_{1}\left(\Phi ;-i \theta_{1}, \ldots,-i \theta_{l}, \zeta_{l+1}, \ldots, \zeta_{p}\right)$ has a limit $\mathscr{G}\left(\Phi ;-i \theta_{1}, \ldots\right.$ $\left.\ldots,-i \theta_{p}, \zeta_{l+1}, \zeta_{p}\right)$ when $\Lambda \rightarrow \infty$ uniformly for $\left(\theta_{1}, \ldots, \theta_{l}\right) \in K^{p}$. 
The main result then follows.

Theorem IV.5. Let the two-body potential be a real even function, continuous outside of the origin (everywhere if $v=1$ ); assume it has the stability property and belongs to $L^{1}\left(\mathbb{R}^{v}\right) \cap L^{2}\left(\mathbb{R}^{v}\right)$.

Then, for a Bose gas at low activity, the following limit exists:

where

$$
\mathscr{G}\left(\Phi ; \zeta^{p}\right)=\lim _{A \rightarrow \infty} \mathscr{G}_{\Lambda}\left(\Phi ; \zeta^{p}\right), \quad \forall \zeta^{p} \in \overline{\mathscr{D}}
$$

$$
\mathscr{G}_{\Lambda}\left(\Phi ; \zeta^{p}\right)=Z_{\Lambda}^{-1} \operatorname{Tr}_{\mathscr{H}_{\Lambda}}\left\{U\left(\varphi_{1}\right) e^{-\left(\zeta_{2}-\zeta_{1}\right) H_{A}} \ldots U\left(\varphi_{p}\right) e^{-\left(\beta+\zeta_{1}-\zeta_{p}\right) H_{A}}\right\}
$$

and

$$
\begin{aligned}
U\left(\varphi_{i}\right) & =\exp i\left\{a\left(\varphi_{i}\right)^{*}+a\left(\varphi_{i}\right)\right\}, \quad \varphi_{\imath} \in L^{2}\left(\Lambda_{0}\right), \quad \Lambda_{0} \leqq \Lambda \\
\mathscr{D} & =\left\{\zeta^{p} \in \mathbb{C}^{p}: \operatorname{Re} \zeta_{1}<\operatorname{Re} \zeta_{2}<\cdots<\operatorname{Re} \zeta_{p}<\beta+\operatorname{Re} \zeta_{1}\right\} .
\end{aligned}
$$

Furthermore, the convergence is uniform on compacts of $\overline{\mathscr{D}}$ and the limit is continuous on $\overline{\mathscr{D}}$.

So we have proved the existence and continuity of the infinite volume limit multitime Green's functions, constructed with exponential operators, and so far all the quasi local algebra (defined as the norm closure of the algebra generated by all $U(\varphi)$ with $\varphi \in L^{2}\left(\mathbb{R}^{v}\right)$ and with compact support).

Acknowledgment. We would like to thank Professor D. Ruelle for calling our attention to this problem and for important remarks.

\title{
References
}

1. For a discussion of this point see J. Ginibre: Some applications of Functional Integration in Statistical Mechanics (Les Houches, Summer 1970).

2. Ruelle,D.: J. Math. Phys. 12, 901 (1971).

3. Bochner, S., Martin, W.I.: Several complex variables, VII, 4, Th. 4. Princeton 1948.

4. Ginibre, J.: J. Math. Phys. 6, 238 (1965).

5. Ruelle,D.: Definition of Green's functions for dilute Fermi gases. Helv. Phys. Acta 45, (2) 215 (1972).

6. Gruber, C.: Thesis, Princeton University, 1968 (Unpublished).

7. Dunford, N., Schwartz, J.: Linear operators, p. 1105, Lemma XI. 9-20. New York: Interscience 1963.

\author{
M. Duneau \\ Centre de Physique Théorique \\ d'Ecole Polytechnique \\ 17 rue Descartes \\ F-75230 Paris Cedex 05/France
}


